\title{
ANÁLISIS DE LA ESTRUCTURA ANATÓMICA DE LA MADERA Y DEL CARBÓN DE DOS ESPECIES DE SAPOTACEAE
}

\section{ANALYSIS OF THE ANATOMICAL STRUCTURE OF WOOD AND CHARCOAL OF TWO SAPOTACEAE SPECIES}

\author{
Graciela Inés Bolzon de Muñiz ${ }^{1, \diamond}$, Ramiro Faria França ${ }^{2}$, Adriano Edis Fiorese ${ }^{2}$, Silvana Nisgoski ${ }^{1}$
}

\begin{abstract}
RESUMEN
El carbón vegetal es un insumo energético muy utilizado en diversos segmentos de la industria siderúrgica, metalúrgica, cimientos y otras, presentando importancia económica e histórica en Brasil. Una de las formas de determinar la procedencia del carbón vegetal es identificando este material, lo que permite comprobar la legalidad de explotación, y cual es su posible origen. Este trabajo tuvo como objetivo describir la anatomía de la madera y del carbón de Pouteria macrophylla (A.DC.) Eyma y Micropholis guianensis (A.DC) Pierre, y comparar las características antes y después del proceso de carbonización, con la finalidad de suministrar más información sobre la estructura de estos materiales. Los cuerpos de prueba se carbonizaron en horno de mufla, a temperatura máxima de $450{ }^{\circ} \mathrm{C}$ durante un período de aproximadamente 6 horas y 30 minutos. La elevada contracción de las células de Pouteria macrophylla y Micropholis guianensis durante el proceso de carbonización causó alteraciones dimensionales, que no perjudicaron el aspecto cualitativo de las características anatómicas, siendo posible la distinción de especies, e identificación del leño carbonizado a través de la descripción de la madera.
\end{abstract}

Palabras clave: Leño carbonizado, estructura anatómica, identificación de especies.

\begin{abstract}
Charcoal is an energetic input much applied in different segments of ironwork, metallurgic, cement industry and others, being important economically and historically in Brazil. One manner of determine the precedence of wood coal is the material identification, what allow to probe the legality of exploration and which is its possible origin. This paper aims to describe wood and charcoal anatomy of Pouteria macrophylla (A.DC.) Eyma and Micropholis guianensis (A.DC) Pierre, and compare the characteristics before and after carbonization process, for the purpose of increase the information about this materials. The samples were carbonized on electric muffle furnace, with maximum temperature of $450^{\circ} \mathrm{C}$, in approximately 6 hours and 30 minutes. The elevate contraction of cells of Pouteria macrophylla and Micropholis guianensis during carbonization process result in dimensional changes, but it not affect qualitative aspects of anatomical characteristics, being possible the distinction of species and identification of charcoal by wood description.
\end{abstract}

Keywords: Carbonized wood, anatomic structure, species identification.

\footnotetext{
${ }^{1}$ Dra. Eng. Florestal. Laboratório de Anatomia e Qualidade da Madeira, Departamento de Engenharia e Tecnologia Florestal,

Universidade Federal do Paraná. 80.210-170,Curitiba, PR, Brasil. silvana.ufpr@gmail.com

${ }^{2}$ Acadêmico de Engenharia Industrial Madeireira. Universidade Federal do Paraná. Curitiba. PR, Brasil.

^Autor para correspondencia: graciela.ufpr@gmail.com

Recibido: 04.10.2012 Aceptado: 26.12.2012
} 


\section{INTRODUCCIÓN}

La mayor parte de la madera producida en Brasil es destinada a la generación de energía, destacándose la producción de carbón vegetal debido a la demanda del sector siderúrgico (Brito 2007), que en los últimos años ha consumido más del $84 \%$ de la producción nacional (Duboc et al. 2007), industria metalúrgica, cimientos y otras, presentando importancia económica e histórica (Dallastra 2010).

El carbón vegetal ha sido utilizado en Brasil desde 1918, con la implantación de la Compañía Siderúrgica Minera. Desde esa época el carbón vegetal siempre ha sido considerado como una materia prima de segunda categoría, de bajo costo, obtenida por actividades secundarias de la tala de bosques nativos (CETEC - MG 1982).

El país aporta aproximadamente el 30\% de la producción mundial, casi en su totalidad para el uso siderúrgico, pero todavía en general, como hace siglos, sin preocupación por el medio ambiente (Colombo et al. 2006). Uno de los problemas asociados a la producción de carbón vegetal en Brasil es el origen de la materia prima. La producción de carbón a partir de bosques implantados ha sido insuficiente para atender la demanda, lo que trajo como consecuencia una mayor presión sobre los remanentes forestales (Duboc et al. 2007).

Aun existiendo sistemas de producción eficientes desde el punto de vista económico, ambiental y social, en la última década se realizaron pocas inversiones en el sector. Sin embargo, con la mayor rigidez de las leyes ambientales y laborales, el aumento de las operaciones conjuntas de organismos ambientales y Policía Federal, se intensificó la demanda de sistemas más modernos para la producción de carbón (Dallastra 2010).

Una de las formas de determinar la procedencia del carbón vegetal es identificando este material, determinando cual es la especie utilizada, la legalidad de su explotación, y su posible lugar de origen. Para la identificación del carbón vegetal se utiliza la antracología, en este caso las muestras de carbón vegetal son comparadas con muestras de madera, o con sus descripciones anatómicas. Diversos trabajos demostraron que las estructuras celulares de la madera y su disposición permanencen semejantes después del proceso de carbonización.

Gonçalves (2010) describió la antracología como una ciencia multidisciplinaria que tiene como principio el análisis y la identificación de carbones, basándose en la anatomía de la madera. En Brasil, los estudios antracológicos también son aplicados con un enfoque conservacionista y tecnológico, como la identificación de especies de procedencia irregular y determinación de la calidad del carbón, pudiendo contribuir en estudios de carbones, de la misma manera que la anatomía de la madera contribuye en diversas áreas.

La xilología es la rama de la ciencia que analiza y describe la disposición de los elementos celulares del leño de los árboles, constituyéndose en una valiosa herramienta para la identificación de especies tropicales (Cury y Tomazello Filho 2011) y conocimiento real de las propiedades de la madera utilizada. Además, para los proveedores es necesario conocer con precisión las especies con las que están trabajando, para por ejemplo adecuar el lote de madera a la legislación vigente para el transporte.

Para este trabajo se escogieron dos especies de la familia Sapotaceae, ya descritas anatómicamente (Kukachka 1982, Detienne y Jacquet 1983) que en Brasil, despierta gran interés por la importancia económica de sus maderas, que frecuentemente son utilizadas en construcción civil, estructuras de tejados, pisos, marcos, fabricación de durmientes, muebles y obtención de láminas para revestimientos (Costa 2006).

Este trabajo tuvo como objetivo describir la anatomía de la madera y del carbón de las especies de Sapotaceae, Pouteria macrophylla (A.DC.) Eyma y Micropholis guianensis (A.DC) Pierre, y comparar las características antes y después del proceso de carbonización, con la finalidad de contribuir con más información sobre la estructura de estas maderas. 


\section{MATERIALES Y MÉTODOS}

Se utilizaron árboles de plantaciones naturales de las especies Pouteria macrophylla (A.DC.) Eyma y Micropholis guianensis (A.DC) Pierre provenientes de un área de la selva amazónica, en el municipio de Nova Maringá, localizado en el norte del Estado de Mato Grosso (Latitud $13^{\circ} 1^{\prime} 2^{\prime \prime}$ Sur, Longitud $57^{\circ} 4^{\prime} 8^{\prime \prime}$ Oeste). Para el análisis, las tortas obtenidas de la región del DAP, con $8 \mathrm{~cm}$ de espesor y diámetro aproximado de $40 \mathrm{~cm}$, se seccionaron en dimensiones menores en el Laboratorio de Anatomía y Calidad de la Madera - LANAQM, de la Universidad Federal do Paraná UFPR. Se confeccionaron cuerpos de prueba orientados en los tres planos de corte de la madera (transversal, radial y tangencial), con dimensiones de $1 \times 1 \times 3 \mathrm{~cm}$, para el análisis de las características anatómicas. Para ablandar los cuerpos de prueba, éstos se hirvieron en agua y cortaron en micrótomo de deslizamiento, con una cuchilla modelo $\mathrm{C}$ para la confección de cortes histológicos, con un espesor de aproximadamente $17 \mu \mathrm{m}$. Posteriormente se colorearon con Safranina y Azul de Astra y, luego se realizó la deshidratación, en una serie alcohólica ascendente. Las secciones se colocaron entre porta y cubreobjeto de vidrio, utilizando Entellán como medio de montaje.

Se obtuvieron imágenes en microscopio Olympus CX-40 con cámara digital Olympus Camedia C-3000 acoplada, y las medidas se realizaron con auxilio del software Olympus MicroSuite TM - Basic.

Para el análisis del carbón, diez cuerpos de prueba de cada especie con $2 \times 2 \times 5 \mathrm{~cm}$, obtenidos en diferentes posiciones radiales, se carbonizaron en horno con control electrónico de micro procesamiento de temperatura, con temperatura máxima de $450^{\circ} \mathrm{C}$, durante un período de aproximadamente 6 horas y 30 minutos.

Las mediciones de los elementos anatómicos del carbón vegetal se realizaron en estereomicroscopio Zeiss Discovery V12 con ayuda del software AxionVision R4.7. También se obtuvieron imágenes de microscopio electrónico de barrido (MEB) con un equipo HITACHI modelo TM-1000, directamente del material macizo.

Fueran realizadas 25 mediciones para poros $/ \mathrm{mm}^{2}$, diámetro de los poros, radios $/ \mathrm{mm}$, altura y ancho del radio, en cada especie, para la madera y el leño carbonizado. Se siguió la norma de la International Association of Wood Anatomists (IAWA 1989) para la descripción de los elementos celulares, y las recomendaciones de Coradin y Muñiz (1991), describiéndose el menor valor medido, la media, el mayor valor, seguido por la desviación estándar calculada. Para el análisis de los valores obtenidos en la caracterización cuantitativa de las muestras, se realizó un análisis de la variancia (ANOVA) a 0,05 de significación y test de Tukey.

La contracción del material fue analizada en cinco muestras, con $2 \times 2 \times 5 \mathrm{~cm}$, orientadas en los planos transversal, radial y tangencial. Fueron medidas la area transversal y las dimensiones radiales y tangenciales antes y después de la carbonización, adaptandose a las recomendaciones de la NBR 7190 (ABNT 1997).

\section{RESULTADOS Y DISCUSIÓN}

\section{Pouteria macrophylla}

La madera de Pouteria macrophylla posee vasos múltiples radiales de 2 a 4, con placas de perforación simples. La porosidad es difusa y se observa la obstrucción de los vasos, ocasionada por aceites y resinas. El diámetro

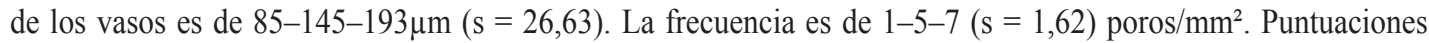
intervasculares alternas, radiovasculares y parénquima-vasculares semejantes. El parénquima axial es en bandas continuas, en formato semejante al reticulado. Los radios son heterogéneos, con células procumbentes y cuadradas en las márgenes, en su mayoría uniseriados, con presencia también de radios biseriados, con 178-424-738 $\mu \mathrm{m}$ (s $=167,75)$ de altura y $15-28-49 \mu \mathrm{m}(\mathrm{s}=8,09)$ de ancho. La frecuencia constatada es de 4-7-11 $(\mathrm{s}=1,98)$ radios por milímetro lineal. 
En el leño carbonizado de Pouteria macrophylla se observan vasos múltiples radiales, sin obstrucción. El

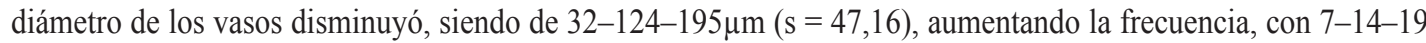
$(\mathrm{s}=2,97)$ poros $/ \mathrm{mm}^{2}$. Las puntuaciones intervasculares alternas y las radiovasculares mantuvieron la estructura. El parénquima axial en líneas continuas, en formato semejante al reticulado, se mantuvo destacable a simple vista. Los radios variaron entre $121-281-1279 \mu \mathrm{m}(\mathrm{s}=228,43)$ de altura y $12-23-37 \mu \mathrm{m}(\mathrm{s}=6,05)$ de ancho. La frecuencia aumentó para 11-13-17 $(\mathrm{s}=1,86)$ radios por milímetro lineal en función de la contracción dimensional.

La variación dimensional de la madera y leño carbonizado de Pouteria macrophylla se presenta en la tabla 1 y las características macro y microscópicas se muestran en las figuras 1 y 2.

Tabla 1. Características de la madera y leño carbonizado de Pouteria macrophylla.

\begin{tabular}{lcccc}
\hline \multirow{2}{*}{\multicolumn{1}{c}{ Característica }} & \multicolumn{2}{c}{ Madera } & \multicolumn{2}{c}{ Carbón } \\
\cline { 2 - 5 } & Media & $\begin{array}{c}\text { Desviación } \\
\text { estándar }\end{array}$ & Media & $\begin{array}{c}\text { Desviación } \\
\text { estándar }\end{array}$ \\
\hline Poros/mm $\mathbf{m}^{\mathbf{2}}$ & $5 \mathrm{a}$ & 1,62 & $14 \mathrm{~b}$ & 2,97 \\
Diámetro de los Poros $(\boldsymbol{\mu m})$ & $145 \mathrm{a}$ & 26,63 & $124 \mathrm{~b}$ & 47,16 \\
Radios/mm & $7 \mathrm{a}$ & 1,98 & $13 \mathrm{~b}$ & 1,86 \\
Altura del Radio $(\boldsymbol{\mu m})$ & $424 \mathrm{a}$ & 167,75 & $281 \mathrm{~b}$ & 135,09 \\
Ancho del Radio $(\boldsymbol{\mu m})$ & $28 \mathrm{a}$ & 8,09 & $23 \mathrm{~b}$ & 6,05 \\
\hline
\end{tabular}

* Medias seguidas por la misma letra en cada característica no difieren estadísticamente por el test de Tukey al $5 \%$ de probabilidad.

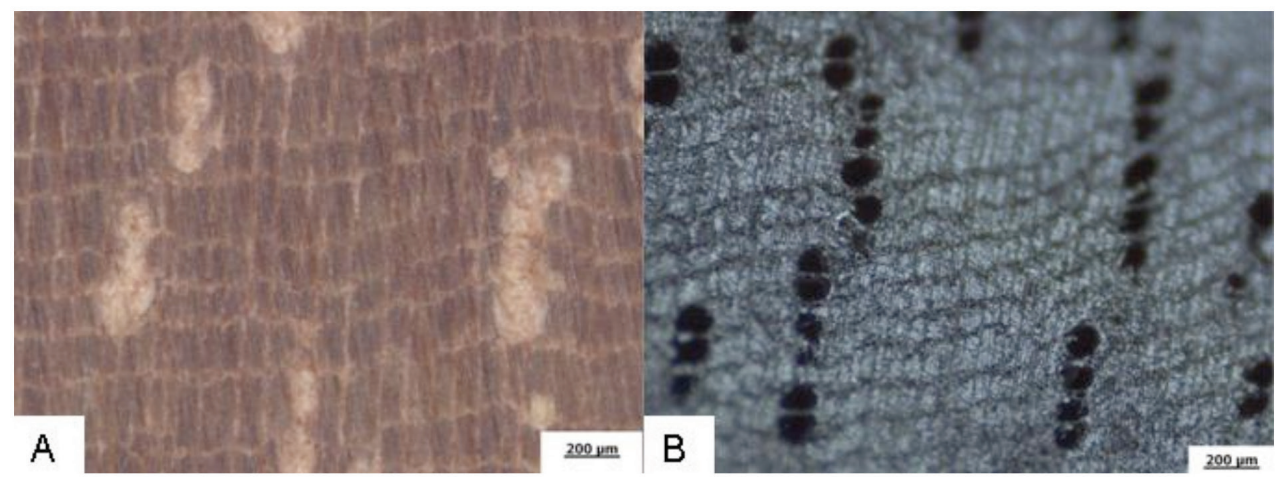

Figura 1. Aspecto macroscópico de la madera (A) y carbón (B) de Pouteria macrophylla. 


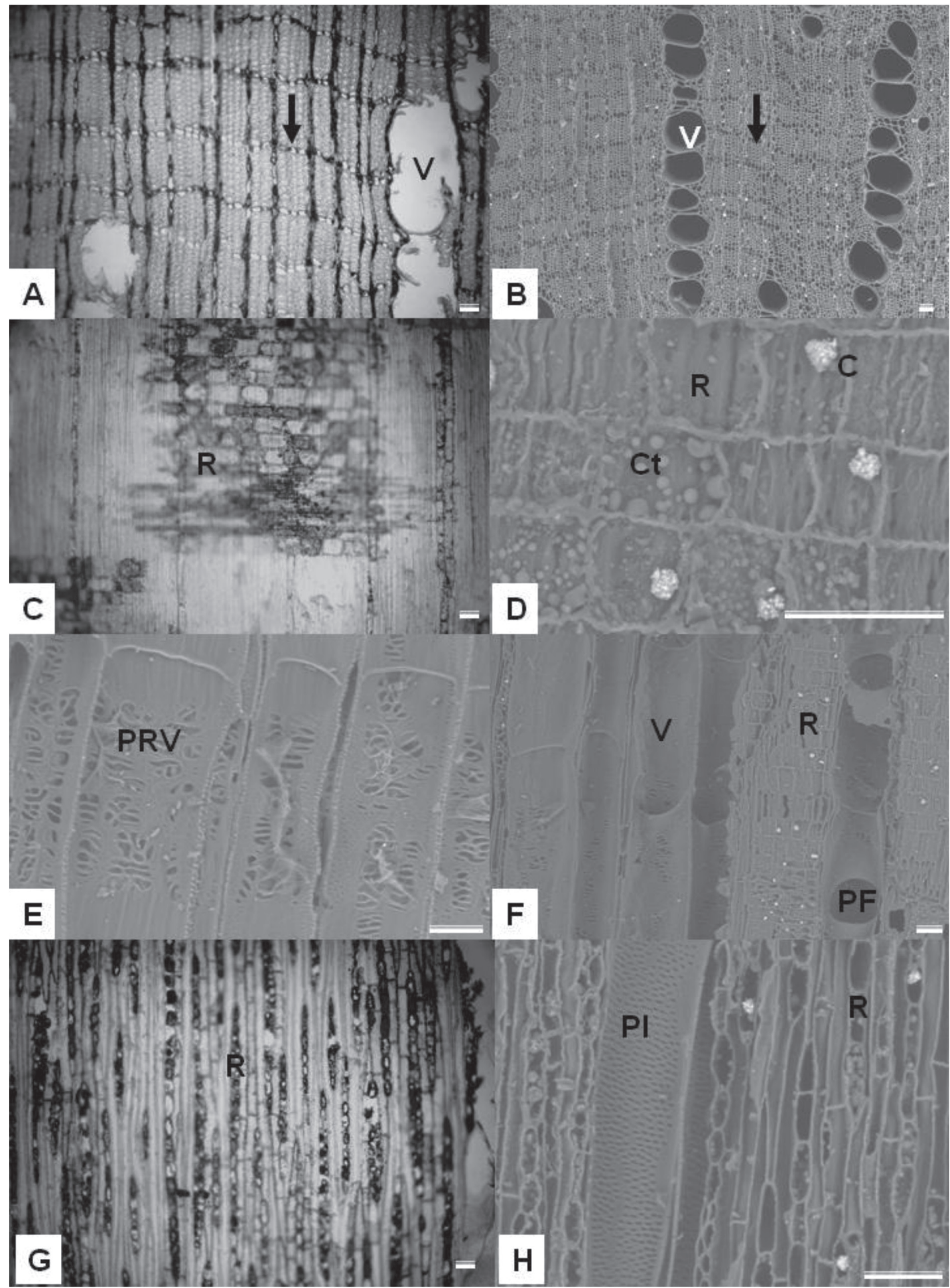

Figura 2. Aspecto microscópico de la madera (A,C,G) y MEB (B,D,E,F,H) del leño carbonizado de Pouteria macrophylla. Flecha: parénquima axial; V: vaso; R: radios; C: cristal; Ct: contenido; PRV: puntuaciones radiovasculares; PF: placa de perforación; PI: puntuaciones intervasculares. Escala: $50 \mu \mathrm{m}$. 
Durante el proceso de carbonización todas las estructuras celulares sufrieron alteraciones dimensionales de manera significativa. El diámetro de los poros disminuyó, aumentando como consecuencia la frecuencia por milímetro cuadrado. Los radios se contrajeron tanto en altura como en ancho, aumentando la cantidad por milímetro lineal.

Resultados semejantes fueron encontrados por Gonçalves (2010) evaluando Pouteria torta, con disminución en el diámetro tangencial de los vasos, y aumento en la frecuencia de los radios. La disminución en altura y ancho de los radios también fue observada en seis especies africanas estudiadas por Prior y Gasson (1993). Muñiz et al. (2012) observaron disminución del ancho del radio en Enterolobium schomburgkii y aumento en Cedrelinga catenaeformis, justificando la variación en función de la diferencia en el espesor de las paredes de las fibras.

Debido a las diferencias significativas observadas en las estructuras anatómicas, se carbonizaron más muestras para el estudio de la contracción de la especie. Se evaluaron los cuerpos de prueba y se compararon sus medidas antes y después de la carbonización adoptada, observándose una disminución del $48 \%$ en el área de la sección transversal de las muestras, con una disminución de $22 \%$ y $32 \%$ en las dimensiones radiales y tangenciales, respectivamente.

Gonçalves et al. (2012) observaron reducción de 19\% en el diámetro tangencial de los vasos de Pouteria torta, $21 \%$ en Dalbergia violaceae y $20 \%$ en Vochysia tucanorum proveniente del cerrado Brasileño, y sugirieron que la abundancia de parénquima puede causar gran contracción volumétrica en el carbón, debido a las paredes delgadas de estas células.

\section{Micropholis guianensis}

La madera de Micropholis guianensis posee vasos múltiples de 2 a 5, solitarios eventuales, con placas de perforación simples. Puntuaciones intervasculares alternas, radio y parénquima-vasculares semejantes. La porosidad

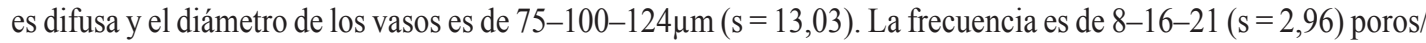
$\mathrm{mm}^{2}$. El parénquima axial es en bandas continuas, con formato similar al reticulado. Los radios son heterogéneos, con células procumbentes y cuadradas, biseriados en casi su totalidad, con 147-274-453 $\mu \mathrm{m}(\mathrm{s}=81,05)$ de altura y $15-22-32 \mu \mathrm{m}(\mathrm{s}=4,80)$ de ancho. La frecuencia constatada es de 4-7-11 $(\mathrm{s}=1,55)$ radios por milímetro lineal.

En el leño carbonizado de Micropholis guianensis se observaron vasos bien definidos, solitarios, geminados y múltiples radiales, con placas de perforación simples sin alteración. Las puntuaciones intervasculares y radiovasculares mantuvieron el aspecto original. El diámetro de los vasos es de $55-92-171 \mu \mathrm{m}(\mathrm{s}=25,34)$. La frecuencia es de 27-49-65 $(\mathrm{s}=8,93)$ poros $/ \mathrm{mm}^{2}$. El parénquima axial es en bandas continuas, con formato semejante al reticulado. Los radios se contrajeron, variando de $95-259-391 \mu \mathrm{m}(\mathrm{s}=81,05)$ de longitud y $11-21-29 \mu \mathrm{m}(\mathrm{s}=5,12)$ de ancho. La frecuencia constatada aumentó, siendo de 8-14-20 $(\mathrm{s}=3,00)$ radios por milímetro lineal.

La variación dimensional de la madera y leño carbonizado de Micropholis guianensis se presenta en la tabla 2 y las características macro y microscópicas se muestran en las figuras 3 y 4. 
Tabla 2. Características de la madera y leño carbonizado de Micropholis guianensis.

\begin{tabular}{ccccc}
\hline \multirow{2}{*}{ Característica } & \multicolumn{2}{c}{ Madera } & \multicolumn{2}{c}{ Carbón } \\
\cline { 2 - 5 } & Media & $\begin{array}{c}\text { Desviación } \\
\text { estándar }\end{array}$ & Media & $\begin{array}{c}\text { Desviación } \\
\text { estándar }\end{array}$ \\
\hline Poros/mm $\mathbf{m m}^{\mathbf{2}}$ & $18 \mathrm{a}$ & 5,35 & $49 \mathrm{~b}$ & 8,93 \\
Diámetro de los Poros $(\boldsymbol{\mu m})$ & $100 \mathrm{a}$ & 13,03 & $92 \mathrm{a}$ & 25,34 \\
Radios/mm & $7 \mathrm{a}$ & 1,55 & $14 \mathrm{~b}$ & 3,00 \\
Altura del Radio $(\boldsymbol{\mu m})$ & $274 \mathrm{a}$ & 81,05 & $259 \mathrm{a}$ & 75,34 \\
Ancho del Radio $(\boldsymbol{\mu m})$ & $22 \mathrm{a}$ & 4,80 & $21 \mathrm{a}$ & 5,12 \\
\hline
\end{tabular}

* Medias seguidas por la misma letra en cada característica no difieren estadísticamente por el Test de Tukey al 5\% de probabilidad.

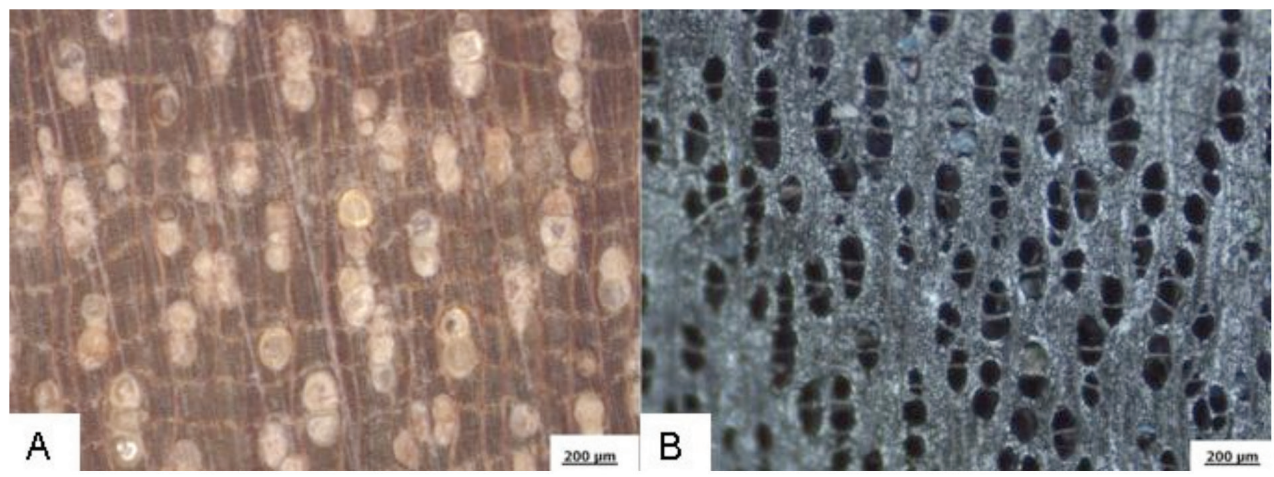

Figura 3. Aspecto macroscópico de madera (A) y carbón (B) de Micropholis guianensis. 


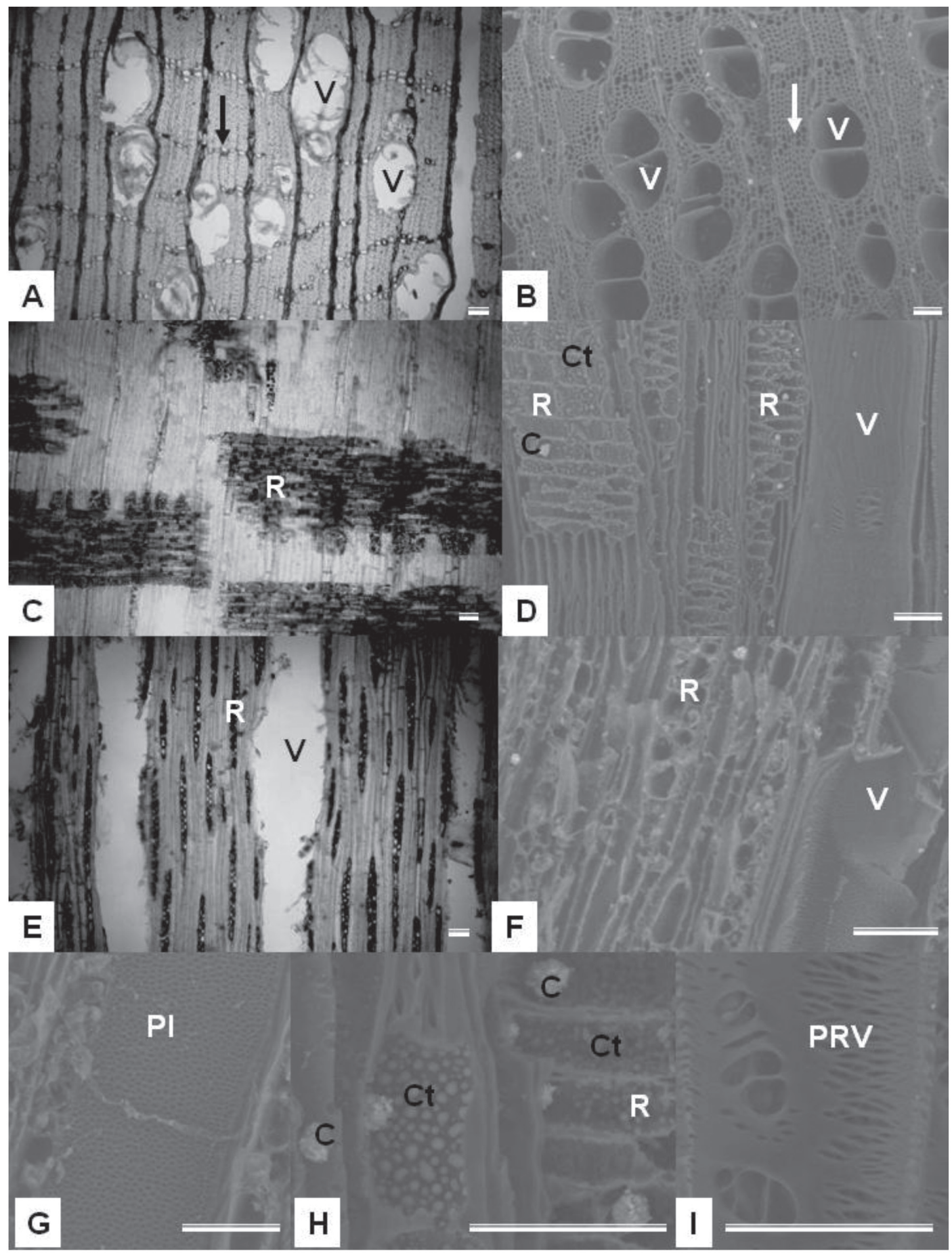

Figura 4. Aspecto microscópico de la madera (A,C,E) y MEB (B,D,F,G,H,I) del leño carbonizado de Micropholis guianensis. Flecha: parénquima axial; V: vaso; R: radios; C: cristal; Ct: contenido; PRV: puntuaciones radiovasculares; PI: puntuaciones intervasculares. Escala: $50 \mu \mathrm{m}$. 
Luego de la carbonización, sólo se observaron variaciones en la frecuencia de poros y radios. Tampoco se observaron alteraciones significativas en el diámetro tangencial de los vasos en Couepia grandiflora, Tapirira guianensis (Gonçalves 2010) e Cedrelinga catenaeformis (Muñiz et al. 2012). El aumento dimensional, pero no significativo en altura de los radios también fue observado por Gonçalves (2010) en especies del cerrado brasileño, en dos especies de Mato Grosso (Muñiz et al. 2012), en oposición a lo reportado en seis especies africanas (Prior y Gasson 1993).

Observando el elevado valor en la frecuencia de poros por milímetro cuadrado medidos en el carbón, se carbonizaron más muestras para el estudio de la contracción de la especie. Se evaluaron los cuerpos de prueba, y compararon sus medidas antes y después de la carbonización. De acuerdo con las mediciones, en la madera de Micropholis guianensis hubo una disminución media de $46 \%$ en el área de la sección transversal de los cuerpos de prueba luego de la carbonización, con una disminución de $21 \%$ y $32 \%$ en las dimensiones radiales y tangenciales, respectivamente.

\section{CONCLUSIÓN}

La frecuencia de poros y radios fue afectada por la carbonización a $450{ }^{\circ} \mathrm{C}$, aumentando en las dos especies estudiadas. Los poros y radios se contrajeron en Pouteria macrophylla y no sufrieron alteraciones significativas en Micropholis guianensis.

La elevada contracción de las células de Pouteria macrophylla y Micropholis guianensis durante el proceso de carbonización causó alteraciones dimensionales, que no perjudicaron el aspecto cualitativo de las características anatómicas, permitiendo la distinción de las especies y la identificación del leño carbonizado por la descripción de la madera.

\section{BIBLIOGRAFÍA}

ABNT. Associação Brasileira de Normas Técnicas. 1997. NBR 7190: Projeto de estruturas de madeira. Anexo B.

Brito, J.O. 2007. O uso energético da madeira. Estudos Avançados 21 (59): 185-193.

CETEC/MG. Fundação Centro Tecnológico de Minas Gerais. 1982. Carvão vegetal; destilação, propriedades e controle de qualidade. Série de Publicações Técnicas 6: 9.

Colombo, S.F.O.; Pimenta, A. S.; Hatakeyama, K. 2006. Produção de carvão vegetal em fornos cilíndricos verticais: um modelo sustentável. XIII SIMPEP. Bauru - SP.

Coradin, V.T.R.; Muniz, G.I.B. 1991. Normas e procedimentos em estudos de anatomia da madeira: I Angiospermae; II - Gimnospermae. IBAMA. Série Técnica n.15. DIRPED. Laboratório de Produtos Florestais - LPF, Brasília.

Cury, G.; Tomazello Filho, M. 2011. Descrição anatômica de espécies de madeira utilizadas na construção civil. Floresta e Ambiente 18 (3): 227-236.

Costa, A.D.C. 2006. Anatomia da madeira em Sapotaceae. Ph.D. Thesis, Instituto de Biociências da Universidade de São Paulo - USP, São Paulo. Brasil. 
Dallastra, E. C. 2010. Sistemas de produção de carvão vegetal existentes no Brasil: Uma análise de viabilidade econômica. MSc. Thesis, Escola de Economia de São Paulo da Fundação Getúlio Vargas - FGV, São Paulo. Brasil.

Detienne, P.; Jacquet, P. 1983. Atlas d'identification des bois de l'amazonie et des regions voiseines. Centre. Technique Forestier Tropical, Nogent s/Marne. 640 pp.

Duboc, E.; Costa, C.J.; Veloso, R.F.; Oliveira, L.S.; Paludo, A. 2007. Panorama atual da produção de carvão vegetal no Brasil e no Cerrado. Documentos EMBRAPA Cerrados. Planaltina, Distrito Federal. Brasil.

Gonçalves, T.A.P. 2010. Anatomia do lenho e do carvão de espécies arbóreas do cerrado no estado de São Paulo, Brasil. MSc. Thesis, Faculdade de Ciências Agronômicas da Universidade Estadual Paulista "Júlio de Mesquita Filho" - UNESP. Botucatu, São Paulo. Brasil.

Gonçalves, T.A.P.; Marcati, C.R.; Scheel-Ybert, R. 2012. The effect of carbonization on wood structure of Dalbergia violaceae, Stryphnodendron polyphyllum, Tapirira guianensis, Vochysia tucanorum and Pouteria torta from the Brazilian cerrado. IAWA Journal 33 (1): 73-90.

IAWA COMMITTE. 1989. IAWA List of microscopic features for hardwood identification. IAWA Bulletin 10 (3): 219-332.

Kukachka, B.F. 1982. Wood anatomy of the neotropical Sapotaceae. XXXII. Richardella. USDAForest Products Laboratory. Research Paper FPL 420.

Muñiz, G.I.B.; Nisgoski, S.; França, R.; Schardosin, F.Z. 2012. Anatomia comparativa da madeira e carvão de Cedrelinga catenaeformis Ducke e Enterolobium schomburgkii Benth. para fins de identificação. Scientia Forestalis 40 (94): 291-297.

Prior, J.; Gasson, P. 1993. Anatomical changes on charring six african hardwoods. IAWA Journal 14 (1): 77-86. 